\title{
Tumor Identification Link Group Identifier
}

National Cancer Institute

\section{Source}

National Cancer Institute. Tumor Identification Link Group Identifier. NCI Thesaurus.

Code C162032.

A sequence of characters used to link multiple tumor identification records to a single finding. 